\title{
Magnetoresistivity modulated response in bichromatic microwave irradiated two dimensional electron systems
}

\author{
J. Iñarrea ${ }^{a}$ \\ Escuela Politécnica Superior, Universidad Carlos III, Leganes, Madrid, 28911, Spain \\ and Instituto de Ciencia de Materiales, CSIC, Cantoblanco, Madrid 28049, Spain \\ G. Platero \\ Instituto de Ciencia de Materiales, CSIC, Cantoblanco, Madrid 28049, Spain
}

(Received 9 July 2006; accepted 12 September 2006; published online 26 October 2006)

\begin{abstract}
The authors analyze the effect of bichromatic microwave irradiation on the magnetoresistivity of a two dimensional electron system. The authors follow the model of microwave driven Larmor orbits in a regime where two different microwave lights with different frequencies are illuminating the sample $\left(w_{1}\right.$ and $\left.w_{2}\right)$. The authors' calculated results demonstrate that now the electronic orbit centers are driven by the superposition of two harmonic oscillatory movements with the frequencies of the microwave sources. As a result the magnetoresistivity response presents modulated pulses in the amplitude with a frequency of $\left(w_{1}-w_{2}\right) / 2$, whereas the main response oscillates with $\left(w_{1}+w_{2}\right) / 2$. (C) 2006 American Institute of Physics. [DOI: 10.1063/1.2364856]
\end{abstract}

In the field of condensed matter physics, very few problems have produced such an intense activity, experimental and theoretical, like microwave induced resistivity oscillations $^{1,2}$ (MIROs) and zero resistance states (ZRSs). ${ }^{3,4}$ From the experimental standpoint, remarkable contributions are being published in a continuous basis. Among them we can highlight activated temperature dependence in the magnetoresistivity $\left(\rho_{x x}\right)$ response, ${ }^{3-5}$ quenching of $\rho_{x x}$ response at high microwave (MW) intensities, ${ }^{2,6}$ absolute negative conductivity and breakdown of $\mathrm{ZRSs},{ }^{2,5-7}$ suppression of MIROs and ZRSs by in-plane magnetic field, ${ }^{8,9}$ and the evidence that MIROs and ZRSs are notably immune to the sense of circular polarization of MW radiation. ${ }^{10}$ Very recently, an experimental achievement has joined the group of new contributions. In this case an ultraclean two dimensional electron system (2DES) is subjected to bichromatic MW radiation coming from two monochromatic sources with different frequencies $w_{1}$ and $w_{2}{ }^{11}$ The unexpected result consists in a $\rho_{x x}$ response which is clearly modulated in the oscillation amplitude. This modulation results to be tunable by increasing intensity of one of the MW sources while keeping the other constant. All these experimental evidences establish real challenges for the theoretical models presented to date. ${ }^{12-20}$ Considering that all these models are not able to achieve consensus about the true origin of these striking phenomena, the new experimental results can be regarded as crucial tests for theories, for the existing ones, and for the ones to come. However, some theoretical contributions are already being presented giving explanation for some of the experimental outcomes. We can stress theoretical proposals for temperature and high MW intensity dependence, ${ }^{21,22} \mathrm{ab}$ solute negative conductivity, ${ }^{23,24} \rho_{x x}$ immunity to the sense of circular polarization of MW radiation, ${ }^{25,26}$ and finally one very recent proposal regarding $\rho_{x x}$ response to bichromatic MW radiation. ${ }^{27}$

In this letter we report a theoretical explanation to the $\rho_{x x}$ modulated amplitude of a 2DES when it is illuminated for two different MW radiations with different frequencies $w_{1}$

${ }^{a)}$ Electronic mail: jesusinarrea@icmm.csic.es and $w_{2}$. In a recently presented model by the authors, ${ }^{19}$ it was demonstrated that in a 2DES subjected to a perpendicular magnetic field and MW radiation, Larmor orbit centers oscillate with the same frequency as the MW field: $M W$ driven Larmor orbits. If we now apply two MW fields to the sample the consequence is that electronic orbit centers are subjected simultaneously to two oscillatory movements with the frequencies of the MW fields $w_{1}$ and $w_{2}$. The outcome is the superposition of both oscillations, giving rise to an oscillatory movement in the orbit center whose amplitude is modulated in the way of pulses. Pulses have the well-known frequency of $\left(w_{1}-w_{2}\right) / 2$, whereas the new oscillatory movement goes with $\left(w_{1}+w_{2}\right) / 2$.

Following the MW driven Larmor orbit model, we first obtain the exact expression of the electronic wave vector for a 2DES in a perpendicular magnetic field $B$ and two MW sources: $:^{19,28,29}$

$$
\begin{aligned}
\Psi(x, t)= & \phi_{n}\left(x-X-x_{\mathrm{cl}}(t), t\right) \exp \left[i \frac{m^{*}}{\hbar} \frac{d x_{\mathrm{cl}}(t)}{d t}\left[x-x_{\mathrm{cl}}(t)\right]\right. \\
& \left.+\frac{i}{\hbar} \int_{0}^{t} L d t^{\prime}\right]\left[\sum _ { m = - \infty } ^ { \infty } J _ { m } [ \frac { e E _ { 1 } X } { \hbar w _ { 1 } } ] \left(\frac{1}{w_{1}}\right.\right. \\
& \left.+\frac{w_{1}}{\sqrt{\left(w_{c}^{2}-w_{1}^{2}\right)^{2}+\gamma^{4}}}\right) e^{i p w_{1} t}+\sum_{n=-\infty}^{\infty} J_{n}\left[\frac{e E_{2} X}{\hbar w_{2}}\right] \\
& \left.\times\left(\frac{1}{w_{2}}+\frac{w_{2}}{\sqrt{\left(w_{c}^{2}-w_{2}^{2}\right)^{2}+\gamma^{4}}}\right) e^{i p w_{2} t}\right],
\end{aligned}
$$

where $\gamma$ is a phenomenologically introduced damping factor for the electronic interaction with acoustic phonons, ${ }^{21} e$ is the electron charge, $\phi_{n}$ is the solution for the Schrödinger equation of the unforced quantum harmonic oscillator, $w_{1}$ and $w_{2}$ are the MW frequencies, $E_{1}$ and $E_{2}$ are the intensities for the MW fields, $w_{c}$ is the cyclotron frequency, $X$ is the center of the orbit for the electron motion, and $x_{\mathrm{cl}}$ is the classical solution of a forced harmonic oscillator driven by two different time dependent forces: 


$$
\begin{aligned}
x_{\mathrm{cl}}(t)=x_{1}(t)+x_{2}(t)= & \frac{e E_{1}}{m^{*} \sqrt{\left(w_{c}^{2}-w_{1}^{2}\right)^{2}+\gamma^{4}}} \cos w_{1} t \\
& +\frac{e E_{2}}{m^{*} \sqrt{\left(w_{c}^{2}-w_{2}^{2}\right)^{2}+\gamma^{4}}} \cos w_{2} t \\
= & A_{1} \cos w_{1} t+A_{2} \cos w_{2} t .
\end{aligned}
$$

$L$ is the classical Lagrangian and $J_{m}$ and $J_{n}$ are Bessel functions. If $w_{1}$ is not very different from $w_{2}$ and the MW field intensities are equal, then we can write $A_{1} \simeq A_{2}=A$ and therefore

$$
\begin{aligned}
x_{\mathrm{cl}}(t) & =A\left[\cos w_{1} t+\cos w_{2} t\right] \\
& =2 A \cos \left[\frac{1}{2}\left(w_{1}-w_{2}\right) t\right] \cos \left[\frac{1}{2}\left(w_{1}+w_{2}\right) t\right],
\end{aligned}
$$

showing that now the oscillatory movement for the Larmor orbit center presents modulated amplitude with a frequency given by $\frac{1}{2}\left(w_{1}-w_{2}\right)$, whereas the main oscillation goes like $\frac{1}{2}\left(w_{1}+w_{2}\right)$.

Now we introduce the scattering suffered by the electrons due to charged impurities randomly distributed in the sample. ${ }^{19,30}$ Following the model described in Ref. 19, firstly we calculate the electron-charged impurity scattering rate $1 / \tau$ ( $\tau$ being the scattering time). Secondly we find the average effective distance advanced by the electron in every scattering jump, that in the case of two MW sources is given by

$$
\Delta X^{\mathrm{MW}}=\Delta X^{0}+A_{1} \cos w_{1} \tau+A_{2} \cos w_{2} \tau,
$$

where $\Delta X^{0}$ is the effective distance advanced when there is no MW field present. Again if $A_{1} \simeq A_{2}=A$ we can write

$$
\Delta X^{\mathrm{MW}}=\Delta X^{0}+2 A \cos \left[\frac{1}{2}\left(w_{1}-w_{2}\right) \tau\right] \cos \left[\frac{1}{2}\left(w_{1}+w_{2}\right) \tau\right] .
$$

Finally the longitudinal conductivity $\sigma_{x x}$ can be calculated: $\sigma_{x x} \propto \int d E\left(\Delta X^{\mathrm{MW}} / \tau\right)\left(f_{i}-f_{f}\right), f_{i}$ and $f_{f}$ being the corresponding distribution functions for the initial and final Landau states, respectively, and $E$ energy. To obtain $\rho_{x x}$ we use the relation $\rho_{x x}=\sigma_{x x} /\left(\sigma_{x x}^{2}+\sigma_{x y}^{2}\right) \simeq \sigma_{x x} / \sigma_{x y}^{2}$, where $\sigma_{x y} \simeq n_{i} e / B$ and $\sigma_{x x}$ $\ll \sigma_{x y}$.

In Fig. 1, we present calculated results for experimental frequencies ${ }^{11}$ (47 and $31 \mathrm{GHz}$ ). In panel (a) we present all the cases (bichromatic and both monochromatic) together for comparison. In the rest of the panels we present each calculated response separately. According to our model the surprising profile obtained for $\rho_{x x}$ response is a reflex of the amplitude modulated oscillatory movement of the Larmor orbit centers, when they are under the influence of both MW fields. In fact, in the experimental graphs ${ }^{11}$ and in the calculated Fig. 1, at least one modulated pulse can be seen. This pulse can also be observed more clearly in Fig. 2, where we present the same situation as in Fig. 1, but with different frequencies (57 and $38 \mathrm{GHz}$ ). Our results are in good agreement with experiments.

In Fig. 3, we present in the bottom graph the calculated bichromatic magnetoresistivity $\rho_{x x}$ as a function of $B$, for experimental frequencies at increasing MW intensity for the case of $47 \mathrm{GHz}$. In the top graph of Fig. 3, we present mathematical functions which simulate the $\rho_{x x}$ behavior for monochromatic and bichromatic driving forces, considering one of them with increasing magnitude. In both graphs it can be observed that a minimum shows up when one of the driving forces is increased. This is because increasing only one Downloaded 12 Mar 2010 to 161.111 .180 .191 . Redistribution subje

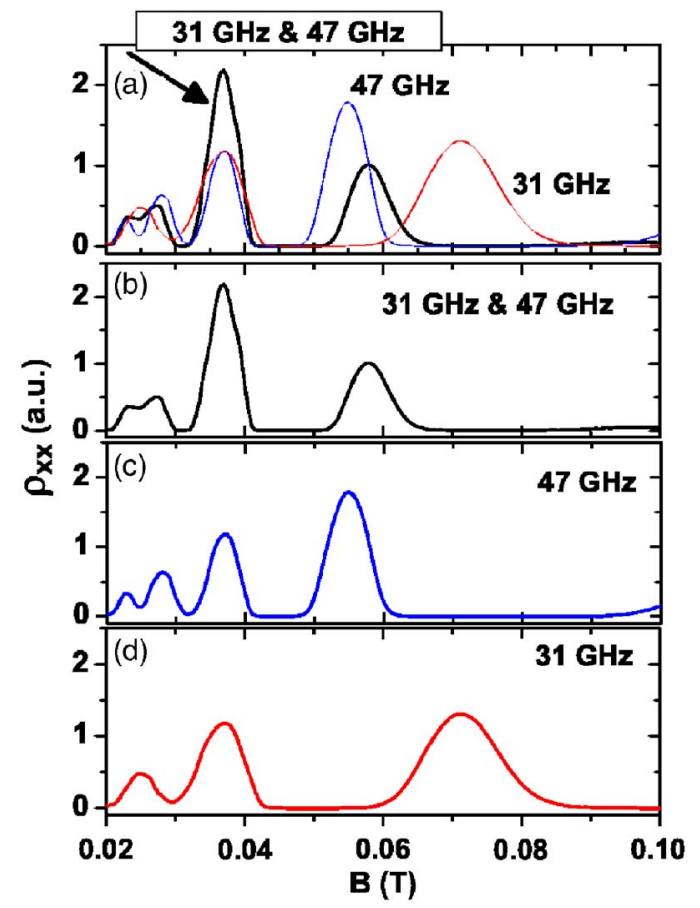

FIG. 1. (Color online) Calculated magnetoresistivity $\rho_{x x}$ as a function of $B$, for experimental MW frequencies (Ref. 11): 47 and $31 \mathrm{GHz}$. (a) Calculated MW responses for all the frequencies considered, monochromatic and bichromatic MW sources, all together for comparison. (b) Bicromatic response (47 and $31 \mathrm{GHz}$ ). (c) Monochromatic, $47 \mathrm{GHz}$. (d) Monochromatic, $31 \mathrm{GHz}$.

of the intensities ( $47 \mathrm{GHz}$ in this case), the bichromatic response will tend to be similar to the monochromatic one. We have considered that, according to our model (see Ref. 19), $\rho_{x x}$ depends on $B$ like $\rho_{x x} \propto B \cos (w \tau)=B \cos \left(S_{c}(w / B)\right)$, where $S_{c}$ is a sample dependent scattering term. In the case of two simultaneously MW driving forces with similar intensities,

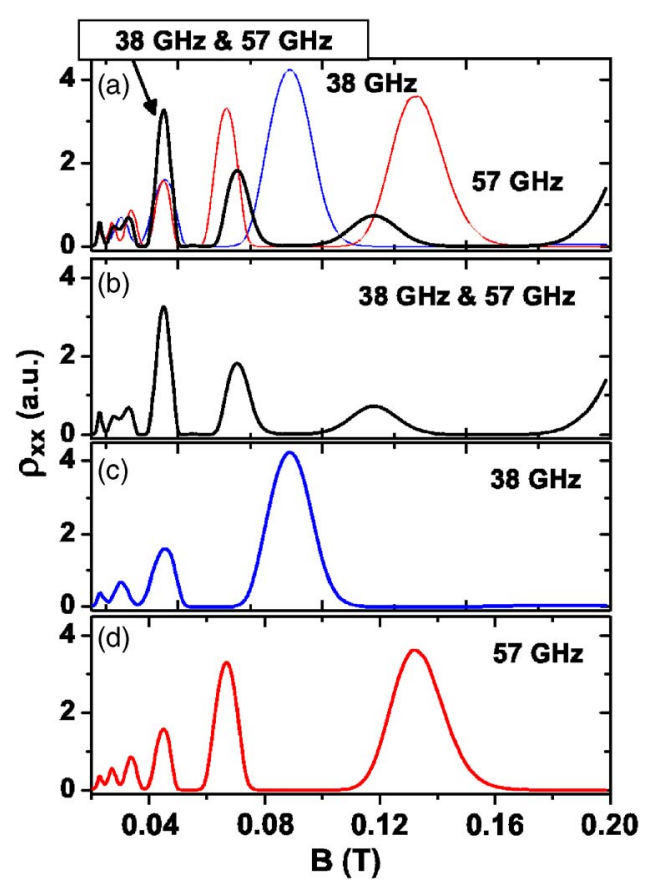

FIG. 2. (Color online) Same as Fig. 1 but for MW frequencies 57 and $38 \mathrm{GHz}$.

to AIP license or copyright; see http://apl.aip.org/apl/copyright.jsp 

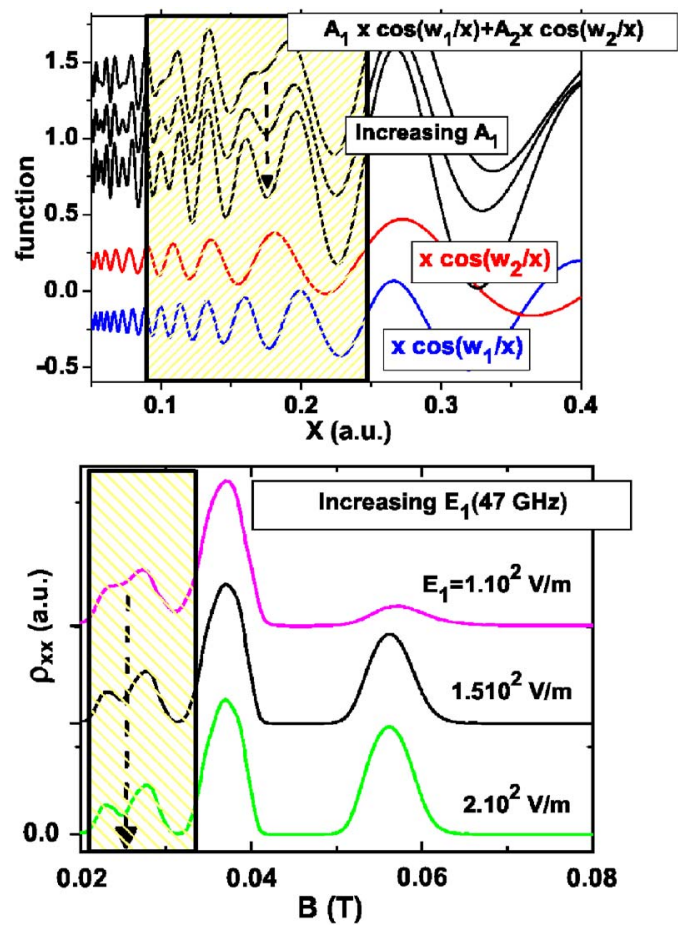

FIG. 3. (Color online) Bottom graph: Calculated magnetoresistivity $\rho_{x x}$ as a function of $B$, for experimental frequencies at increasing MW intensity for the $47 \mathrm{GHz}$ MW source. Top graph: Mathematical functions to simulate $\rho_{x x}$ behavior for monochromatic and bichromatic driving forces, considering one of them with increasing magnitude.

$$
\rho_{x x} \propto B \cos \left[\frac{1}{2} \frac{\left(w_{1}-w_{2}\right)}{B} S_{c}\right] \cos \left[\frac{1}{2} \frac{\left(w_{1}+w_{2}\right)}{B} S_{c}\right] .
$$

Considering the last expression we have tested our current theory using the experimental data (Fig. 2 of Ref. 11). The aim is to eventually obtain the experimental frequencies used (47 and $31 \mathrm{GHz}$ ). In order to do that first we have measured in the experimental figure the $B^{-1}$ periodicity in monochromatic and bichromatic graphs and also the width of the corresponding bichromatic pulse. Once this information was obtained and using our expressions for the $\rho_{x x}$ dependence with $B$ we have been able to reach numerical values for the pulse and main oscillation frequencies. For the pulse we have obtained a value of $9.3 \mathrm{GHZ}$, and for the main frequency $38.9 \mathrm{GHz}$. Comparing these values with the ones obtained directly from the experimental frequencies, (47 -31) $\mathrm{GHz} / 2=8 \mathrm{GHz}$ for the pulse and $(47+31) \mathrm{GHz} / 2$ $=39 \mathrm{GHz}$ for the main frequency, we can see that the agreement finally achieved is quite reasonable.

In conclusion we have demonstrated that the experimental results ${ }^{11}$ regarding the $\rho_{x x}$ modulated response of a $2 \mathrm{DES}$ subjected to a $B$ and bichromatic MW can be explained in terms of the electronic orbit centers being driven by the superposition of two harmonic oscillatory movements with the frequencies of the microwave sources.

This work was supported by the MCYT (Spain) Grant No. MAT2005-06444, the "Ramon y Cajal" program (J.I.), and the EU Human Potential Programme No. HPRN-CT2000-00144.

${ }^{1}$ M. A. Zudov, R. R. Du, J. A. Simmons, and J. L. Reno, Phys. Rev. B 64, 201311 (2001).

${ }^{2}$ S. A. Studenikin, M. Potemski, A. Sachrajda, M. Hilke, L. N. Pfeiffer, and K. W. West, Phys. Rev. B 71, 245313 (2005); S. A. Studenikin, M. Potemski, P. T. Coleridge, A. Sachrajda, and Z. R. Wasilewski, Solid State Commun. 129, 341 (2004).

${ }^{3}$ R. G. Mani, J. H. Smet, K. von Klitzing, V. Narayanamurti, W. B. Johnson, and V. Umansky, Nature (London) 420, 646 (2002).

${ }^{4}$ M. A. Zudov, R. R. Du, N. Pfeiffer, and K. W. West, Phys. Rev. Lett. 90, 046807 (2003).

${ }^{5}$ R. L. Willett, L. N. Pfeiffer, and K. W. West, Phys. Rev. Lett. 93, 026804 (2004).

${ }^{6}$ R. G. Mani, V. Narayanamurti, K. von Klitzing, J. H. Smet, W. B. Johnson, and V. Umansky, Phys. Rev. B 69, 161306(R) (2004); R. G. Mani, Appl. Phys. Lett. 85, 4962 (2004); Physica E (Amsterdam) 22, 1 (2004).

${ }^{7}$ M. A. Zudov, R. R. Du, N. Pfeiffer, and K. W. West, Phys. Rev. B 73, 041303 (2006)

${ }^{8}$ C. L. Yang, R. R. Du, L. N. Pfeiffer, and K. W. West, Phys. Rev. B 74, 045315 (2006).

${ }^{9}$ R. G. Mani, Phys. Rev. B 72, 075327 (2005).

${ }^{10}$ J. H. Smet, B. Gorshunov, C. Jiang, L. Pfeiffer, K. West, V. Umansky, M. Dressel, R. Dressel, R. Meisels, F. Kuchar, and K. von Klitzing, Phys. Rev. Lett. 95, 116804 (2005).

${ }^{11}$ M. A. Zudov, R. R. Du, N. Pfeiffer, and K. W. West, Phys. Rev. Lett. 96, 236804 (2006).

${ }^{12}$ A. C. Durst, S. Sachdev, N. Read, and S. M. Girvin, Phys. Rev. Lett. 91, 086803 (2003).

${ }^{13}$ C. Joas, J. Dietel, and F. von Oppen, Phys. Rev. B 72, 165323 (2005).

${ }^{14}$ X. L. Lei and S. Y. Liu, Phys. Rev. Lett. 91, 226805 (2003).

${ }^{15}$ V. Ryzhii and V. Vyurkov, Phys. Rev. B 68, 165406 (2003); V. Ryzhii, ibid. 68, 193402 (2003); V. Ryzhii and R. Suris, J. Phys.: Condens. Matter 15, 6855 (2003); V. Ryzhii, R. A. Suris, and B. S. Shchamkhalova, Sov. Phys. Semicond. 20, 1299 (1986).

${ }^{16}$ P. H. Rivera and P. A. Schulz, Phys. Rev. B 70, 075314 (2004).

${ }^{17}$ Junren Shi and X. C. Xie, Phys. Rev. Lett. 91, 086801 (2003).

${ }^{18}$ A. V. Andreev, I. L. Aleiner, and A. J. Millis, Phys. Rev. Lett. 91, 056803 (2003).

${ }^{19}$ J. Iñarrea and G. Platero, Phys. Rev. Lett. 94, 016806 (2005).

${ }^{20}$ I. A. Dimitriev, M. G. Vavilov, I. L. Aleiner, A. D. Mirlin, and D. G. Polyakov, Phys. Rev. B 71, 115316 (2005).

${ }^{21}$ J. Iñarrea and G. Platero, Phys. Rev. B 72, 193414 (2005).

${ }^{22}$ X. L. Lei and S. Y. Liu, Phys. Rev. B 72, 075345 (2005).

${ }^{23}$ J. Iñarrea and G. Platero, Appl. Phys. Lett. 89, 052109 (2006).

${ }^{24}$ Kang-Hun Ahn, J. Korean Phys. Soc. 47, 666 (2005).

${ }^{25}$ J. Dietel, Phys. Rev. B 73, 125350 (2006).

${ }^{26}$ G. Gumbs, Phys. Rev. B 72, 125342 (2005).

${ }^{27}$ X. L. Lei, Phys. Rev. B 73, 235322 (2006).

${ }^{28}$ E. H. Kerner, Can. J. Phys. 36, 371 (1958).

${ }^{29}$ K. Park, Phys. Rev. B 69, 201301(R) (2004).

${ }^{30}$ B. K. Ridley, Quantum Processes in Semiconductors, 4th ed. (Oxford University Press, Oxford, 1993), p. 282. 